\title{
Computed tomography angiography for renal artery stenosis in children: a flip flop isn't always bad
}

\author{
James S. Donaldson ${ }^{1}$
}

Received: 28 August 2020 / Revised: 28 August 2020 / Accepted: 6 October 2020 / Published online: 17 October 2020

(C) Springer-Verlag GmbH Germany, part of Springer Nature 2020

In this issue of Pediatric Radiology, Orman et al. [1] present their experience with Computed Tomography Angiography (CTA) for renal artery stenosis in children. I commend the authors for evaluating CTA and digital subtraction angiography (DSA) and helping clarify the indications for each. There has long been suspicion that CTA is not adequate as a screening or diagnostic tool. The authors have shown that CTA had a sensitivity of $90.0 \%$ and specificity of $89.7 \%$ for the diagnosis of renal artery stenosis (RAS). They added that CTA diagnosed all cases of main renal artery stenosis - an important point. The lesions that were missed were in smaller intra-renal vessels.

My opinion of the value of CTA for RAS has evolved over time. As an interventional radiologist, I've performed many conventional angiograms in children for RAS. I've learned that some lesions can be extremely subtle and that even with today's CTA, some lesions cannot be detected on CTA. This limitation was pointed out by the authors, with two third-order arterial lesions in their study not detected by CTA but later found on a DSA study. I recall the early days of CTA when merely seeing the main renal arteries was considered a success - and my opinion was that so many lesions would be missed that CTA for RAS was a wasted effort.

In time, however, CT resolution has remarkably improved. Combined with pediatric-trained staff, well-designed protocols and close radiologist oversight, most renal vascular lesions can now be detected, as the authors have shown. The authors didn't stress enough that the CTA examinations in their study were likely as good as they get, performed at Texas Children's Hospital, one of the largest children's

James S. Donaldson

jdonaldson@luriechildrens.org

1 Department of Medical Imaging,

Ann \& Robert H. Lurie Children's Hospital of Chicago,

225 E. Chicago Ave., Chicago, IL 60614, USA hospitals in North America. There should have been a disclaimer - don't try this at home - a warning for radiologists who don't see many children and might not have equipment or staff to pull off high-quality exams. The authors did make the point that one of their false-positive cases was a misinterpretation of noisy images resulting from a low-radiation-dose exam. These studies need the highest resolution possible; it is not the time to go super low dose!

I disagree with one of the authors' comments that in patients highly likely to have RAS because of risk factors or their clinical presentation, CTA might be unnecessary and these patients can proceed directly to DSA and appropriate intervention. I would argue that a CTA should now be performed prior to any DSA. There is value in knowing the positive CTA findings while planning for DSA and intervention - rather than the old-school approach, "we'll find out if we need to treat when we get there." Scheduling, staffing, materials, correct arterial access, and a thorough discussion with the family about the procedure are all important, and knowing them in advance can help an intervention go more smoothly. There are also situations where from the CTA we clearly see the vascular abnormality but recognize that there is no appropriate endovascular treatment. In these patients, an unnecessary invasive DSA examination can be avoided altogether.

There is still a question that needs to be addressed before steering every child with hypertension to a CTA. What does the pediatrician do with a negative CTA result, recognizing there are some lesions that cannot be detected? I believe the answer is this: the same thing the pediatrician does when he or she first diagnoses hypertension in a new patient. They run through the differential diagnosis of pediatric hypertension and use their clinical judgment to determine whether imaging is warranted at all - don't forget there is a huge denominator of children with hypertension who never have imaging. There are clinical scenarios where yes, regardless of negative US and CTA results, a DSA is probably warranted. But in many children without such compelling clinical pictures, a negative US or negative CTA might suffice to stop the imaging workup. 
The authors' statement that during the study 103 patients had negative CTA and did not need to undergo DSA because their "hypertension resolved completely or a cause of hypertension other than RAS was found" might be a little overstated. It's entirely possible that some of those patients continue to be hypertensive and some might even have an undiagnosed renovascular cause. Given the findings of this study, the combination of high-quality CTA with clinical input and good judgment lead me to believe that that number of missed cases is probably very small.

This paper has contributed to my evolving stance from believing that CTA for RAS is a wasted effort to believing that CTA can play a valuable role in the pediatric patient with hypertension. In an election season, flip-flopping one's opinion is seldom viewed positively. Let's just say my views have matured!

\section{Compliance with ethical standards}

Conflicts of interest None

\section{Reference}

1. Orman G, Masand PM, Kukreja KU et al (2020) Diagnostic sensitivity and specificity of CT angiography for renal artery stenosis in children. Pediatr Radiol. https://doi.org/10.1007/ s00247-020-04852-5

Publisher's note Springer Nature remains neutral with regard to jurisdictional claims in published maps and institutional affiliations. 